\title{
High-dose intravenous methylprednisolone for the prophylactic treatment of cluster headache
}

\author{
Sanami Kawada*, Kenichi Kashihara, Takaki Imamura and Manabu Ohno
}

\begin{abstract}
Background: Triptans are effective for immediate relief of episodic cluster headache $(\mathrm{CH})$ but do not reduce the frequency of attacks. Intravenous bolus injection of corticosteroids like methylprednisolone (MP) has been reported to decrease the frequency of $\mathrm{CH}$ attacks. We validated the prophylactic efficacy of MP pulse therapy by monitoring $\mathrm{CH}$ recurrence over several years following treatment of six consecutive male patients (mean age: 38.8 years, range: 26-54 years) afflicted by frequent (often daily) $\mathrm{CH}$ attacks.

Findings: Total MP dose per infusion was 250-500 mg for five patients and $125 \mathrm{mg}$ for the sixth (a diabetic). Highdose MP was administered for 2 or 3 consecutive days in hospital for the first two patients treated. The next four patients received a single bolus injection at presentation, and in some cases a second injection days later at an outpatient clinic. The first two cases treated were also prescribed daily oral prednisolone for at most 6 months while the latter four cases were not. The frequency of $\mathrm{CH}$ attacks was markedly reduced in all patients, with intervals between attacks ranging from 4 to 23 months. We noted no apparent adverse events following MP administration.
\end{abstract}

Conclusions: High-dose MP therapy reduced $\mathrm{CH}$ attack frequency and improved patient quality of life.

Keywords: Cluster headache, Methylprednisolone, Steroid pulse therapy

\section{Introduction}

Subcutaneous injection of sumatriptan succinate is an effective treatment for the immediate relief of episodic cluster headache $(\mathrm{CH})$ but does not reduce the frequency of future attacks. Prolonged or frequent attacks and associated treatment visits can greatly impair patient quality of life. In Japan, self-administration of sumatriptan succinate was approved in February 2008, and this change has greatly reduced emergency visits and hospital admissions due to $\mathrm{CH}$. However, patients with chronic $\mathrm{CH}$ may still require frequent hospital visits, taxing resources and increasing personal medical expenses. To reduce the frequency of attacks, guidelines from the Japanese Society of Neurology recommend calcium channel blockers (verapamil, lomerizine hydrochloride), glucocorticoids, or ergotamine tartrate. Calcium channel blockers are often useful in the prevention of migraine headache, but are less effective in the prevention of cluster headaches. Oral glucocorticoids are often effective against

\footnotetext{
* Correspondence: kawada@kyokuto.or.jp

Department of Neurology, Okayama Kyokuto Hospital, 567-1 Kurata, Naka-ku, Okayama 703-8265, Japan
}

\section{Springer}

$\mathrm{CH}$ : however, the side effects may be intolerable for some patients. Several recent studies have reported the benefits of intravenous bolus injection of methylprednisolone (MP) for episodic $\mathrm{CH}$. In this case series, we evaluated the efficacy of this treatment for Japanese male patients experiencing frequent $\mathrm{CH}$ attacks.

\section{Material and methods}

This case series reports the clinical results for six consecutive male patients with frequent episodic $\mathrm{CH}$ treated by bolus injection of MP. MP was administered by intravenous (i.v.) drip over two hours up to a total dose of 250-500 mg per infusion with the exception of a diabetic patient who was administered $125 \mathrm{mg}$ MP per infusion. If the patient experienced a severe $\mathrm{CH}$ attack at presentation, they were injected subcutaneously with sumatriptan succinate before MP injection. The first two cases in the series were admitted to hospital and received MP pulse therapy for 2 or 3 consecutive days as inpatients. If $\mathrm{CH}$ was not suppressed, a second course of pulse therapy was administered after a four-day interval. The latter four patients could not be admitted due to 
work or domestic circumstances, and thus were administered a single bolus MP injection and in some cases, a second bolus injection at a later time in an outpatient department (Table 1). This study was approved by The Ethics Committee of Okayama Kyokuto Hospital and informed consent was obtained from all subjects prior to the study.

\section{Results}

At presentation, all six patients reported daily $\mathrm{CH}$ attacks that required subcutaneous injection of sumatriptan succinate and (or) inhalation of pure oxygen. These therapies were effective, but the frequent attacks seriously disrupted quality of life. The MP pulse therapy regimens used on initial presentation, the times of recurrence, and oral prednisolone regimens are presented in Table. In all cases, $\mathrm{CH}$ attack frequency was markedly reduced following MP therapy and no patient reported severe $\mathrm{CH}$ for at least four months after treatment. Case 1 relapsed thrice and was treated as an inpatient except on the fourth occasion. Case 2 reported no headache for two days after the first trial of MP pulse administration (one injection per day for three consecutive days): however, he suffered from severe headache again on the sixth day of admission and was administered another series of injections. Case 2 relapsed only twice, although 3 or 4 non-consecutive high-dose bolus injections were required on each recurrence. The other four cases were never hospitalized. Three of these four patients relapsed only once $(6,7$, and 11 months after initial treatment) and were treated by a single bolus injection of MP each time. Case 4 relapsed four times, but also required only a single bolus injection each time, and each treatment required only a half-day absence from work. We noted no adverse events following steroid pulse therapy, such as gastrointestinal bleeding or psychiatric symptoms, and the diabetic patient (Case 5) demonstrated no loss of glucose control.

\section{Discussion}

Cluster headaches afflict $0.07-0.09 \%$ of the population, and the incidence is 9.8 events/million person-years Swanson et al. (1994). The prevalence of $\mathrm{CH}$ is 5-9 times higher in men, and indeed all patients in this case series were men. Cluster headache is a rare disease but symptom severity precludes placebo-controlled studies. Therefore, therapeutic regimens are inconsistent across institutions and countries. According to the Japanese guidelines for chronic headache, triptans or oxygen inhalation is the first-line therapy for acute $\mathrm{CH}$ attacks, and most patients respond well to either treatment. However, it is recommended that inhalation of $100 \%$ oxygen be initiated within 10 minutes of attack onset, making this treatment option impractical for patients with unpredictable $\mathrm{CH}$. Subcutaneous sumatriptan injection is effective and Japanese approval of self-injection

Table 1 Cluster headache attacks and steroid theraphy

\begin{tabular}{|c|c|c|c|c|c|c|}
\hline Case & Age & Sex & $\begin{array}{l}\text { Intervals between } \mathrm{CH} \text { attacks } \\
\text { (months) }\end{array}$ & $\begin{array}{l}\text { Methylprednisolone dose } \\
\text { (intravenous) }\end{array}$ & Admission & $\begin{array}{c}\text { Prednisolone dose and duration } \\
\text { (daily oral) }\end{array}$ \\
\hline \multirow[t]{4}{*}{1} & 54 & Male & Initial presentation & 250 mg (Days 1 and 2) & Yes & 10 mg, 90 days \\
\hline & & & 4 & 250 mg (Days 1-3) & Yes & none \\
\hline & & & 4 & 250 mg (Days 1 and 2) & Yes & 5 mg, 60 days \\
\hline & & & 5 & 250 mg (Day 1) & No & 5 mg, 180 days \\
\hline \multirow[t]{3}{*}{2} & 33 & Male & Initial presentation & 250 mg (Days 1-3, 7-9) & Yes & 20 mg, 10 days \\
\hline & & & 23 & 250 mg (Days 1 and 9) + 500 mg (Day 4) & No & 15 mg, 30 days \\
\hline & & & 11 & 500 mg (Days 1, 7, 11, and 13) & No & 10 mg, 40 days \\
\hline \multirow[t]{2}{*}{3} & 33 & Male & Initial presentation & 250 mg (Day 1) + 500 mg (Day 18) & No & none \\
\hline & & & 6 & 250 mg (Day 1) & No & none \\
\hline \multirow[t]{5}{*}{4} & 38 & Male & Initial presentation & 250 mg (Day 1) & No & none \\
\hline & & & 11 & 250 mg (Day 1) & No & none \\
\hline & & & 7 & 250 mg (Day 1) & No & none \\
\hline & & & 5 & 250 mg (Day 1) & No & none \\
\hline & & & 7 & 250 mg (Day 1) & No & none \\
\hline \multirow[t]{2}{*}{5} & 49 & Male & Initial presentation & 125 mg (Days 1 and 44) & No & none \\
\hline & & & 7 & 125 mg (Day 1) & No & none \\
\hline \multirow[t]{2}{*}{6} & 26 & Male & Initial presentation & 500 mg (Day 1) & No & none \\
\hline & & & 11 & 500 mg (Day 1) & No & none \\
\hline
\end{tabular}


(December 2007) has reduced hospital visits due to $\mathrm{CH}$. Intranasal sumatriptan and oral zolmitriptan (intranasal zolmitriptan is not yet approved in Japan) are also effective for patients that cannot self-inject sumatriptan. Intranasal lidocaine or cocaine is also effective but rarely used in Japan.

During active $\mathrm{CH}$ periods, severe headaches occur almost daily, interfering with work or school and presenting patients with a significant financial burden for drugs or oxygen. Prophylactic therapy would thus greatly improve quality of life, relieve financial hardship, and save medical resources. The guidelines from the Japanese Society of Neurology recommend ergotamine, calciumchannel blockers, or corticosteroids for prophylaxis at the beginning of a cluster period, although the efficacy of these treatments is highly variable. Double-blind trials have demonstrated the effectiveness of verapamil, but bradycardia and heart block are serious risks. Although lomerizine has less severe cardiovascular side effects and is useful for migraine, there is currently no evidence that lomerizine is an effective prophylactic treatment for $\mathrm{CH}$. Moreover, there are no double-blind placebo-controlled studies of corticosteroid efficacy: nonetheless, corticosteroids have been used for rapid $\mathrm{CH}$ control for more than 60 years Horton (1952). Oral and intravenous corticosteroids may be beneficial Ashkenazi \& Schwedt (2011); Shapiro (2005). For oral administration, daily 40-80 mg prednisolone with gradual reduction over 10 days or longer is recommended Couch \& Ziegler (1978).

There are few reports on the efficacy of intravenous steroid infusion Reija et al. (1998); Cianchetti et al. (1998); Mir et al. (2003); Antonaci et al. (2005). Reija et al. (1998) reported a series of 18 episodic $\mathrm{CH}$ patients treated with $16 \mathrm{mg}$ dexamethasone (i.v.) tapering off after 15 days. On this cohort, 17 patients showed reduced $\mathrm{CH}$ attack frequency Reija et al. (1998). Cianchetti et al. (1998) reported a single case with chronic $\mathrm{CH}$ who showed no response to two weeks of daily $100 \mathrm{mg}$ prednisone but responded dramatically to single $500 \mathrm{mg}$ doses of intravenous MP Cianchetti et al. (1998). Mir et al. (2003) treated 14 episodic $\mathrm{CH}$ patients with $250 \mathrm{mg}$ intravenous $\mathrm{MP}$ on three consecutive days, followed by $90 \mathrm{mg}$ oral prednisone with gradual tapering over 4 weeks, and reported superior results compared to previous treatments Mir et al. (2003). Finally, Antonaci et al. (2005) treated 13 episodic $\mathrm{CH}$ patients with $30 \mathrm{mg} / \mathrm{kg}$ intravenous $\mathrm{MP}$ on the eighth day of the active $\mathrm{CH}$ period and reported significantly reduced attack frequency in all patients, whereas three patients experienced complete headache remission Antonaci et al. (2005).

The therapeutic benefits of corticosteroids may stem, at least in part, from anti-inflammatory effects because inflammation may play an important role in the onset and exacerbation of CH Hannerz (1991). Orbital phlebography and gallium single photon emission computerized tomography revealed inflammation in the cavernous sinus ipsilateral to the $\mathrm{CH}$ pain side during the attack Gawel et al. (1990); Hardebo \& Ryberg (1989). Moreover, elevated serum concentrations of some inflammatory mediators such as haptoglobin, orosomucoid, and interleukin-I beta, have been reported during $\mathrm{CH}$ attacks Hannerz (1991); Martelletti et al. (1993). Several investigations have reported elevated baseline plasma cortisol levels during $\mathrm{CH}$ attacks Waldenlind et al. (1987); Leone et al. (1995); Strittmatter et al. (1996). In addition to cortisol, testosterone and melatonin may also be linked to the pathogenesis of CH Stillman (2006). Positron emission tomography revealed hyperactivation in the gray matter of the ipsilateral hypothalamus during the acute pain state May et al. (1998); Sprenger et al. (2004). Hypothalamic activation coupled with elevated serum cortisol suggests that episodic $\mathrm{CH}$ patients may have a dysfunctional hypothalamicpituitary-adrenal axis. Recently, hypothalamic deep brain stimulation (hDBS) has been used effectively to treat $\mathrm{CH}$ patients Magis et al. (2012). The therapeutic mechanism of hDBS may be similar to high-dose MP injection, suppression of hypothalamic hyperactivity by negative feedback.

High-dose MP injection may produce serious side effects, including infectious diseases resulting from immunosuppression, hypertension, and elevated blood glucose: therefore, this treatment option should be used with caution (particularly as outpatient therapy) in the elderly or for patients with serious underlying diseases such as diabetes. However, MP is far less invasive than hDBS and so should be considered before hDBS. Moreover, younger patients and those without serious contraindication rarely exhibit serious side effects in response to high-dose MP. Finally, MP pulse therapy can be administered rapidly with little need for school or work absence. Thus, high-dose MP injection may be an optimal treatment for otherwise healthy active $\mathrm{CH}$ patients.

\section{Competing interests \\ The authors declare that they have no competing interests.}

\section{Authors' contributions}

KK designed the study. All authors recruited patients, and collected data. SK drafted the report. KK contributed to the review and revision of the report. All authors read and approved the final manuscript.

Received: 21 December 2012 Accepted: 10 March 2013 Published: 11 April 2013

\section{References}

Antonaci F, Costa A, Candeloro E, Sjaastad O, Nappi G (2005) Single high-dose steroid treatment in episodic cluster headache. Cephalalgia 25:290-295 Ashkenazi A, Schwedt T (2011) Cluster headache - acute and prophylactic therapy. Headache 51:272-286

Cianchetti C, Zudeas A, Marchei F (1998) High-dose intravenous methylprednisolone in cluster headache. J Neurol Nuerosug Psych 64:418 Couch JR, Jr, Ziegler DK (1978) Prednisone therapy for cluster headache. Headache 18:219-221 
Gawel MJ, Krajewski A, Luo YM, Ichise M (1990) The cluster diathesis. Headache 30:652-655

Hannerz J (1991) Orbital phlebography and signs of inflammation in episodic and chronic cluster headache. Headache 31:540-542

Hardebo JE, Ryberg B (1989) CSF findings in cluster headache indicative of inflammatory reaction or disease. Cephalalgia 9(suppl 10):301-302

Horton BT (1952) Histaminic cephalgia. J Lancet 72:92-98

Leone M, Lucini V, D'Amico D, Moschiano F, Maltempo C, Fraschini F, Bussone G (1995) Twenty-four-hour melatonin and cortisol plasma levels in relation to timing of cluster headache. Cephalalgia 15:224-229

Magis D, Jensen R, Schoenen J (2012) Neurostimulation therapies for primary headache disorders: present and future. Curr Opin Neurol 25:269-76

Martelletti P, Granata M, Giacovazzo M (1993) Serum interleukin-l beta is increased in cluster headache. Cephalalgia 13:343-345

May A, Bahra A, Buchel C, Frackowiak RSJ, Goadsby PJ (1998) Hypothalamic activation in cluster headache attacks. Lancet 352:275-278

Mir P, Alberca R, Navarro A, Montes E, Martinez E, Franco E, Cayuela A, Lozano P (2003) Prophylactic treatment of episodic cluster headache with intravenous bolus of methylprednisolone. Neurol Sci 24:318-321

Reija G, Nider G, Koscica N, Negro C, Musco G (1998) Dexamethasone in the treatment of episodic cluster headaches: an open study of 18 patients. Funct Neurol 13:171-172

Shapiro RE (2005) Corticosteroid treatment in cluster headache: Evidence, rationale, and practice. Curr Pain Headache Rep 9:126-131

Sprenger T, Boecker H, Tolle TR, Bussone G, May A, Leone M (2004) Specific hypothalamic activation during a spontaneous cluster headache attack. Neurology 62:516-517

Stillman M (2006) Steroid hormones in cluster headaches. Curr Pain Heacache Rep 10:147-52

Strittmatter M, Hamann GF, Grauer M, Fischer C, Blaes F, Hoffmann KH, Schimrigk $\mathrm{K}$ (1996) Altered activity of the sympathetic nervous system and changes in the balance of hypophyseal, pituitary, and adrenal hormones in patients with cluster headache. Neuroreport 7:1229-1234

Swanson JW, Yanagihara T, Stang PE, O'Fallon WM, Beard CM, Melton L, 3rd, Guess HA (1994) Incidence of cluster headaches: a population-base study in Olmsted County, Minnesota. Neurology 44:433-437

Waldenlind E, Gustafsson SA, Ekbom K, Wetterberg L (1987) Circadian secretion of cortisol and melatonin in cluster headache during active cluster periods and remission. J Neurol Neurosurg Psychiatry 50:207-213

doi:10.1186/2193-1801-2-156

Cite this article as: Kawada et al:: High-dose intravenous methylprednisolone for the prophylactic treatment of cluster headache. SpringerPlus 2013 2:156.

\section{Submit your manuscript to a SpringerOpen ${ }^{\circ}$ journal and benefit from:}

- Convenient online submission

- Rigorous peer review

- Immediate publication on acceptance

- Open access: articles freely available online

- High visibility within the field

- Retaining the copyright to your article

Submit your next manuscript at $\gg$ springeropen.com 